\title{
Cloud based VoIP Application in Aircraft Data Networks
}

\author{
Amrutha $^{1}$, R. Thandeeswaran ${ }^{2}$ and N. Jeyanthi ${ }^{3}$ \\ School of Information Technology and Engineering \\ VIT University, Vellore, Tamil Nadu, India \\ 1amrutha.2013@vit.ac.in, ${ }^{3}$ njeyanthi@vit.ac.in
}

\begin{abstract}
There has been a high increase in air traffic for the past ten years and to attract more customers many in-flight facilities are introduced by the carriers. These include Video-OnDemand, use of Personal Digital Assistants (PDA) etc. To provide these facilities the VoIP technology is used more often. But maintaining VoIP infrastructure along with the Aircraft Data Network equipment's will be more cumbersome for the carriers, along with the added infrastructure cost. Thus an improvised architecture has been proposed which helps carriers provide these facilities to the customers in a cost effective and reliable manner. Most of the aircraft communications to the ground rely on satellite for providing these services to the customers. The proposed method uses satellite communication along with cloud based VoIP to provide an efficient quality of service to the passengers. The ground stations used for satellite communication would be connected to a high speed Storage Area Network, to which the cloud network on which the VoIP application is running is also connected. So that whenever a passenger on the flight sends a message/data to the VoIP application first it would be send to the ground stations via the satellite which is then accessed through the SAN server, thus in turn providing faster, reliable and efficient communication to the customers.
\end{abstract}

Keywords: Voice Over IP (VoIP), Aircraft Data Network(ADN),Cloud Computing, Storage Area Network (SAN)

\section{Introduction}

In the past couple of years the number of people opting air travel have increased four times the previous years. This has led to increased competition among the air-carriers to attract more customers. Flight carriers are adopting different advanced technologies to provide better in-flight facilities to the passengers thus making the travel more enjoyable and memorable. Apart from the regular internet services provided other facilities like Video-On-Demand, gamming services, permitting passengers to use their own Personal Digital assistants (PDA) are some of the added features now commonly seen in many flights [1]. With the existing Internet services, Voice over IP (VoIP) technology can be used to provide the same existing facilities in more efficient way. For example the use of VoIP based softphone can used to make calls instead of passengers own PDA. As this would provide better call quality as well would be cost efficient also. Satellite communication is one of the most reliable and efficient communication technology. Usually the flight communication from the pilot's cockpit (when in air) to air-traffic controllers is done using the radio signals. This communication is important because important details like flight's current status, route updates (if any) is communicated using this method. But nowadays flight carriers use satellite technology along with the radio communication for better status update of the flights current position. So using satellite technology flight carriers can also provide the VoIP based in-flight facilities in a 
more reliable way with high Quality of Service (QoS). A typical scenario of this communication is shown in the below figure (Figure 1). Where the data from the flight is send in the form of a signal to the satellite. This data is then amplified and send to the ground station on another frequency (The change of frequency is done so that the ground station distinguishes between the send and received frequency and the amplification of the signal is important as it has to travel several kilometres before it reaches the ground station).

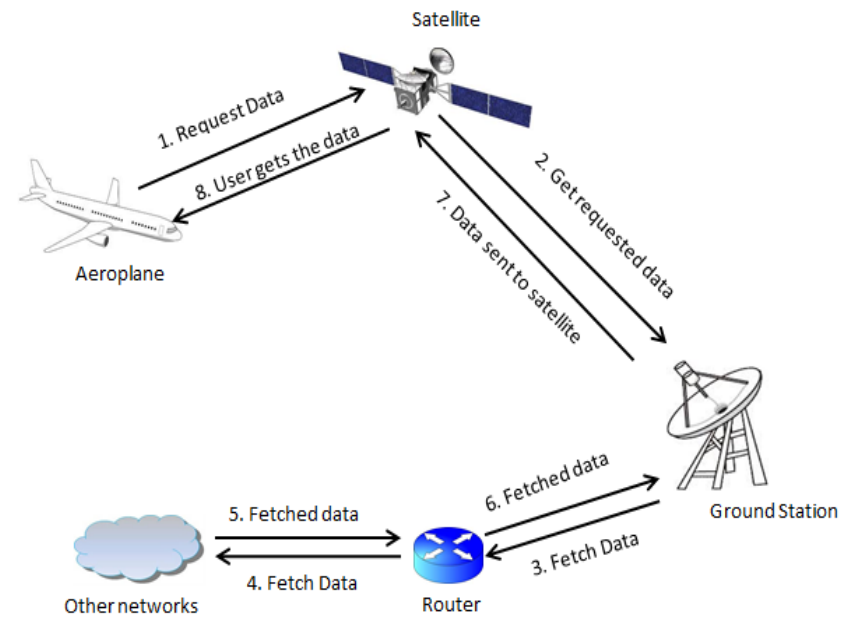

Figure 1. Fight Communication Using Satellite Technology

VoIP application requires additional infrastructure like terminals, gateways, gatekeepers, multi point control units (MCU) for its working. Adding these components to the existing Aircraft Data Network in the flight would incur additional costs to the carriers. With the emerging development of cloud computing, business are able to reduce a lot of infrastructure cost. So here also to reduce the cloud computing technology is found as the best solution to run the VoIP application with less cost and more efficiency.

\subsection{Different VoIP Protocols}

There are different VoIP protocols that can be selected based on the type of communication. Some of the commonly used protocols are the Session Initiated Protocol (SIP), H.323 and Skinny Client Control Protocol (SCCP).

1.2.1. Session Initiated Protocol (SIP): SIP works in the Application layer of the OSI model. It is a protocol that is used for setting up a call between the communicating devices and managing and terminating the session. It does not perform any other tasks like encoding or compression of the data. Every entity interacting/ using SIP is called a User Agent. User Agents can be client based or server based or both. User Agent Client (UAC) is involved in sending requests to the server and the User Agent Server (UAS) processes these requests. UAC and UAS are the only components of the SIP.

1.2.2. H.323: It is a protocol used for communicating multimedia data, which support real-time transfer of audio/video data over the packet networks. The main components of H.323 are terminals, gateway, gatekeepers and multipoint control units (MCU).Terminals are standard devices (like personal computer, mobile devices etc) that 
is capable of running multimedia application. Gateways are mostly routers that connects H.323 device network with the non H.323 network.

Gatekeepers provide addressing, authentication and authorization for the terminals or gateways. Management of bandwidth utilization is also its responsibility. MCU supports conference of more than three H.323 terminals.

1.2.3. Skinny Client Control Protocol (SCCP): SCCP is a session signalling light weighted protocol that is used with Cisco Unified Communications Manager. It is Cisco proprietary software and is used in communication between the IP phones and Cisco Call Manager.

The paper proposes an architecture where in the VoIP application can be used without the installation of added infrastructure. The diagrammatic representation of the architecture is shown in Figure 2 and a detailed description of the architecture and working has been described in the following sections.

\section{Proposed Architecture}

The architecture proposed is referred to as Cloud based VoIP ADN Architecture (C-VoIP ADNA) . Whenever a data is requested from the flight it is send to the satellite first. The satellite then sends this data signal to its ground station on the earth. The nearest ground station that can receive the signal receives the data signal and sends it to the VoIP application running on the cloud (that is also connected to the SAN). Once the data is fetched from the cloud, it is send back to the SAN from where the ground station receives it. The ground station now sends the fetched data via the uplink channel to the satellite and then it reaches the passenger.

All the ground stations are connected to the Storage Area Network (SAN) using a high speed link. The VoIP service that is running on the cloud is also connected to the SAN. So that whenever ground station receives the data from the satellite, it can be send to the cloud using the high speed SAN network. Figure 2 shows a diagrammatic representation of the proposed architecture.

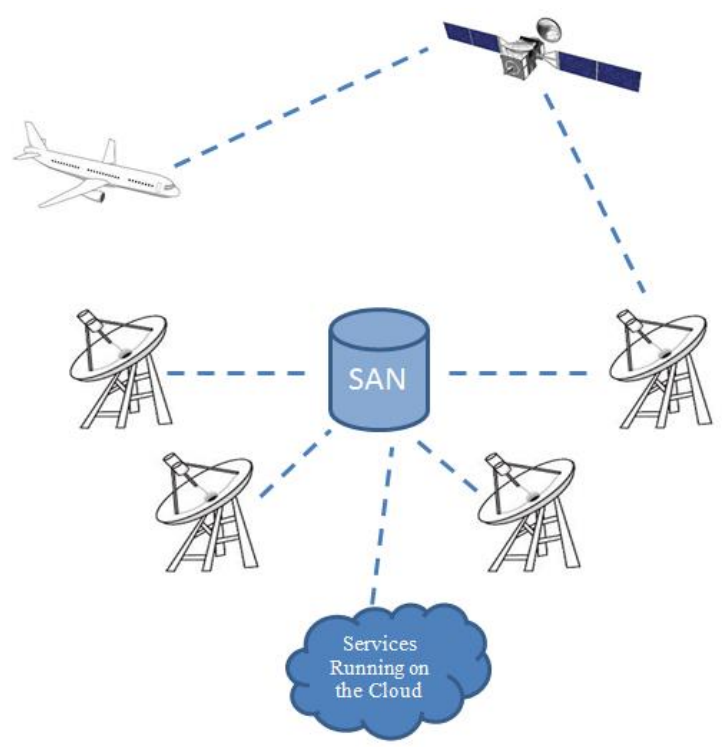

Figure 2. C-VoIP ADNA 
2.1. VoIP Service: Voice Over IP (VoIP) implementation requires added infrastructure for routing the voice data through the Internet. In flight the VoIP data is routed through the Internet (in the proposed schema the internet access is achieved via satellite communication).The voice data generated is first encapsulated into any one of the voice payload protocols like RTP, this is then encapsulated into the UDP-IP packet and routed through the internet. Figure 3 shows the block representation of a single VoIP packet.

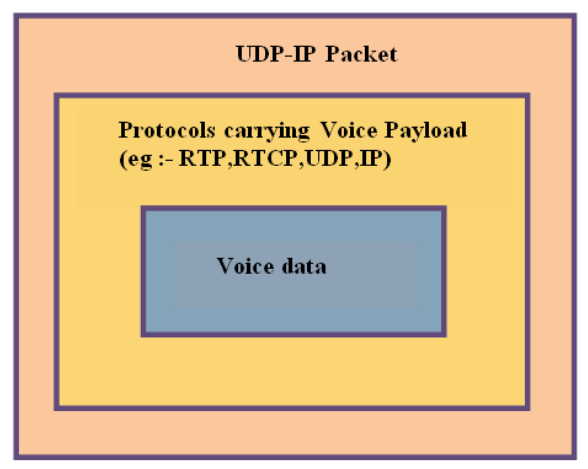

Figure 3. Sample VolP Packet

Voice data generated when we talk is in the form of analog signals, this analog signal has to be converted to digital signal and is transmitted through a packet switched network. Since voice data consumes more bandwidth than the normal data, the data produced should be compressed using any codec standards (eg., G.723.1 standard). This compressed data is segmented (as one packet cannot carry the entire data) and is send to the call manager. Call manger on receiving this data finds an appropriate gatekeeper to route the information to the destination.

VoIP uses two protocols for communication; one is the call control and signalling protocols and the other is voice payload carrying protocols. Table 1 has a summarized list of some of the commonly used signalling and speech transmission protocols in VoIP along with commonly used codec standards.

Table 1. VolP Protocols and Codec Standards

\begin{tabular}{|c|c|c|}
\hline $\begin{array}{c}\text { VoIP Signalling } \\
\text { Protocol }\end{array}$ & $\begin{array}{c}\text { VoIP Speech } \\
\text { Transmission } \\
\text { Protocol }\end{array}$ & $\begin{array}{c}\text { Codec } \\
\text { Standards }\end{array}$ \\
\hline H.323 & $\begin{array}{c}\text { Real Time Transport } \\
\text { Protocol (RTP) }\end{array}$ & G.723.1 \\
\hline H.248 & $\begin{array}{c}\text { Real Time Transport } \\
\text { Control Protocol } \\
\text { (RTCP) }\end{array}$ & G.711a \\
\hline $\begin{array}{c}\text { Session Initiated } \\
\text { Protocol(SIP) }\end{array}$ & UDP & G.711u \\
\hline $\begin{array}{l}\text { Skinny Client } \\
\text { Control } \\
\text { Protocol(SCCP) }\end{array}$ & & G.729a \\
\hline
\end{tabular}


2.2. Cloud VoIP Service: Running VoIP application requires installation of additional VoIP infrastructure (i.e., call manager and a separate network to transfer voice data) would add an extra overhead to the existing operational cost of airlines. So the C-VoIP ADNA uses cloud infrastructure service where the VoIP application will be running, which can be accessed via the SAN.

There are many languages/development kits that can be used to implement the VoIP application like Asterisk, SoftSwitch, BlueBox etc. The architecture shown in Figure 4 is of an Asterisk based VoIP application deployed in the cloud which is connected to the SAN network, to which the ground stations are also connected. Asterisk consists of various VoIP protocols that can be used for communication along with drivers that are used for maintaining compatibility while communication. Another component part of Asterisk is the codec that is used for compression of voice data. Different commonly used codec standards are listed in Table 1.

2.3. Communication in Storage Area Network: A SAN is a dedicated high performance network to move data between heterogeneous servers and storage resources. A separate dedicated network is provided which avoids any traffic conflicts between clients and servers on the traditional messaging network [5].

A SAN basically consists of three logical layers namely; Host layer, Fabric layer and the Storage layer. Host layer mainly comprises of the Host Bus Adapter (HBA) which performs the data lookup and retrieval tasks thus reducing the processing tasks on the server and providing faster data access. Next layer is the Fabric layer which consists of fiber cables that are used for accessing the storage devices connected to the SAN. These cables are connected via switches since the distance travelled by them is long. Storage layer consists of storage arrays, disks that are used to storage purpose. These storage devices are not like the commonly seen devices, it has some added intelligence included in them also thus playing an important role in the performance of the SAN.The protocols used for communication in SAN also plays an important role in achieving the high performance. There are two types of protocols used in a SAN communication, one is the Fibre Channel Protocols and the other is the Small Computer System Interface (SCSI) protocol. The Fibre Channel Protocols are used by the SAN hardware to communicate and the SCSI is used by the software applications to communicate with the hard drives.

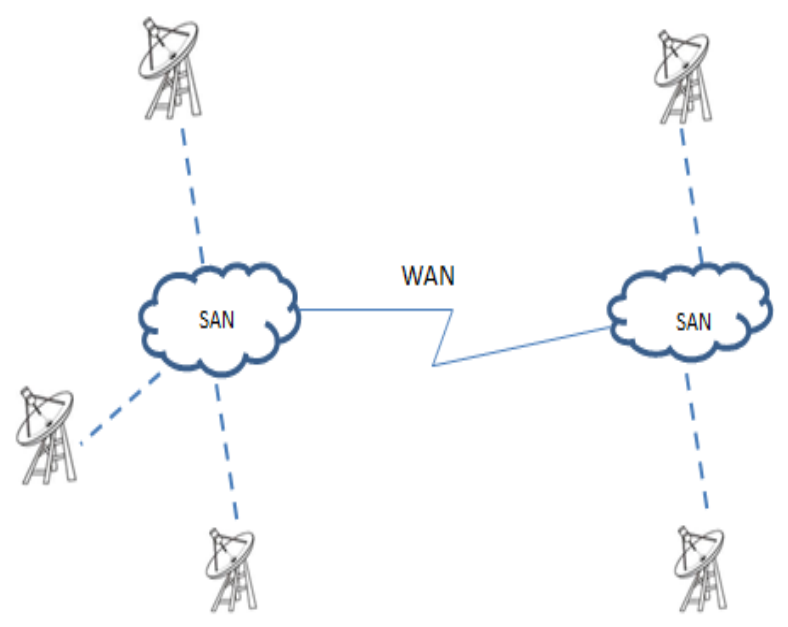

Figure 4. Multiple SAN's Connected via WAN 
A Fibre Channel based SAN combines the high performance of an I/O channel and the advantages of a network (connectivity and distance of a network) using similar network technology components like routers, switches and gateways [5].

In C-VoIP ADNA if the performance gets affected by connecting all the ground stations (that are very far) to SAN, another efficient method would be to group a few ground stations under one SAN and the other under another SAN. Now the two SAN's are interconnected using Wide Area Network (WAN). Figure 4 shows a small scenario how multiple SAN's can be connected via WAN.

\subsection{Cloud Based VoIP Application}

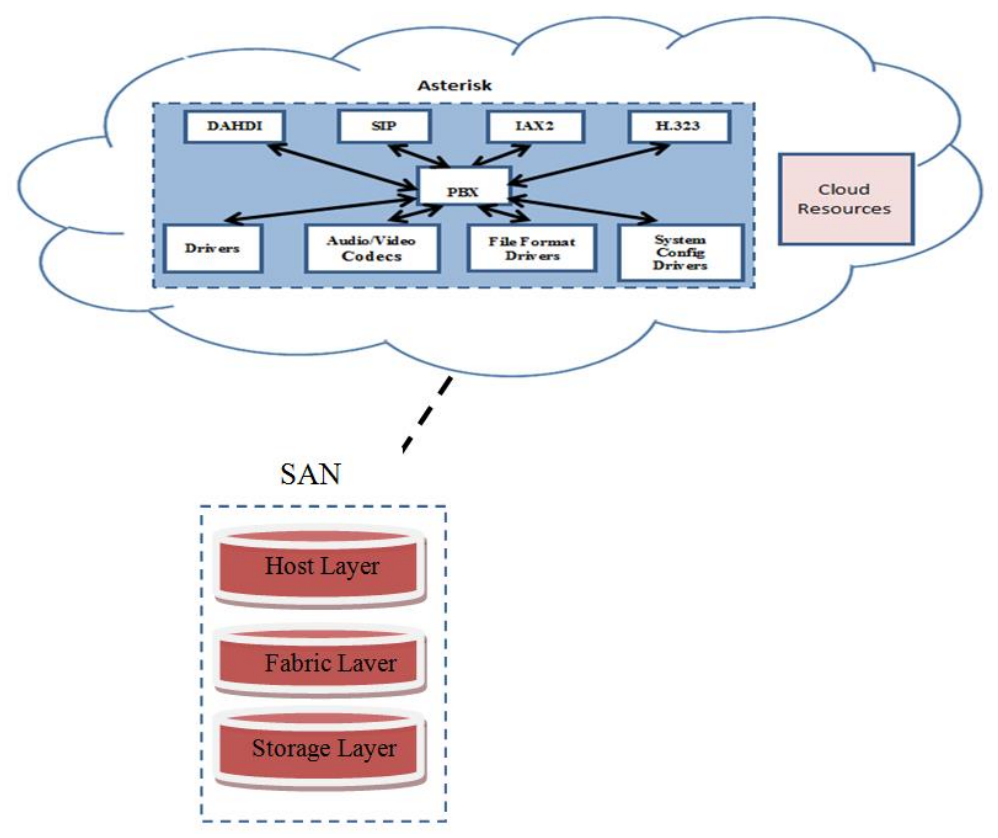

Figure 5. Architecture of Cloud based VolP

\section{Working of C-VoIP ADNA}

The customers interested in using the VoIP services would access them using the software user interface provided in the aircraft. When a request is made using the interface, the data is then send to the satellite. The satellite then modulates this signal and sends it to the ground station on the earth using another frequency. The ground station receiving this data sends it to the SAN server which can be access by the VoIP application running on the cloud. The flight position is monitored by a handshake mechanism. The ground stations sends a polling message to the satellite, this is then relayed to the aircraft. The aircraft responds thereby completing the handshake. The polling message is send at regular intervals to keep track of the fight's position. Every communication involves sending a unique code with it that can be used to identify the communicator. For example when the satellite sends a signal to the ground station, it would contain a unique code embedded into it. This allows the ground station to confirm the identity of the satellite it is communicating with. 
3.1. Security Analysis of C-VoIP ADNA: Every device/application in network should be secured from unauthorized access. With the rise in number of security breaches into networks, making networks fully secured is becoming more difficult.

C-VoIP ADNA uses four main technologies; satellite, cloud computing, VoIP, high speed SAN. Each technology has its own security issues associated with it, below is a brief analysis of the security issues that need to be looked into while deploying this architecture.

The security of the VoIP application is to an extent depended on the how secured is the cloud network. Security of the cloud depends on the type of cloud the application is running on. Basically cloud provides three types of deployment models, namely:-

1. Public model: Security is provided by third party

2. Private model: Security is provided by the individual.

3. Hybrid model: Security can be provided by both third Party and individual.

Controlling and monitoring ground station networks are a challenging task. An attacker can try to gain access to the network that the ground station connects to. But since the ground stations are directly connected to SAN server, only authorised requests would be able to gain access to the network. SAN is an important network component in the proposed architecture and it should be protected from unauthorized access. A strategy that can be used is to group one or more server/switches/storage devices in a zone. This logical grouping will prevent access of device in one zone with the other (if it doesn't possess sufficient access permission). Zoning can be done on ground stations also which would help segregate the ground stations into zones, thus preventing unwanted access.

\section{Conclusion}

In-flight services provided to the passengers are gaining more importance as more and more passengers are opting for airlines that provide these over the others (that do not provide them). VoIP services is a more preferred technology because of the high QoS achieved as it can send the voice data over the IP network at less cost.

Cloud computing is an emerging technology that has been revolutionizing the way people connect and compute. Cloud based VoIP can change the way the in-flight services are provided to the customers. C-VoIP ADNA is an architecture that helps to achieve high speed reliable communication, keeping the cost to very minimal that can be achieved in air. The architecture combines the best technologies to provide high quality service to passengers making their air journey highly satisfying.

\section{References}

[1] M. Luglio, C. Roseti and F. Zampognaro, "TCP Noordwijk+: An upgrade for a cost effective cloud computing via satellite", (2013).

[2] R. Kumar Khattar, M. S. Murphy, G. John Tarella and K. E. Nyst, "Introduction to Storage Area Network", International Technical Support Organization, Available: www.redbooks.ibm.com, (1999).

[3] R. Buyya, C. S. Yeo, S. Venugopal, J. Broberg and I. Brandic, "Cloud computing and emerging IT platforms: Vision, hype, and reality for delivering computing as the 5th utility", Future Generation Computer Systems, vol. 25, (2009), pp. 599-616.

[4] N. Thanthry, M. S. Ali and R. Pendse, "Security, Internet Connectivity and Aircraft Data Networks", IEEE Magazine of Security, Internet Connectivity and Aircraft Data Networks, vol. 21, (2006), pp. 251-255.

[5] P. Sarkar, K. Voruganti, K. Meth, O. Biran and J. Satran, "Internet Protocol storage area networks", IBM Systems Journal, vol. 42, (2003).

[6] R. Mahindru, R. Sarkar and M. Viswanathan, "Software defined unified monitoring and management of clouds", IBM Journal of Research and Development, vol. 58, (2014). 
[7] Z. Xin-ying, T. Xiao-dong and M. Lu-peng, "Zoning Implementations in Storage Area Networks", International Conference on Challenges in Environmental Science and Computer Engineering (CESCE), Whuan, China, (2010) March 6-7.

[8] F. B. Shaikh and S. Haider, "Security threats in cloud computing", International Conference for Internet Technology and Secured Transactions (ICITST), Abu Dhabi, (2011) December 11-14.

[9] Y. Amanatullah, C. Lim, H. P. Ipung and A. Juliandri, "Toward cloud computing reference architecture: Cloud service management perspective", International Conference on ICT for Smart Society (ICISS), Jakarta, (2013) June 13-14.

[10] P. Kalagiakos and P. Karampelas, "Cloud Computing learning", Application of Information and Communication Technologies (AICT), (2011).

[11] Bhatia,AShah NThanthry and R. Pendse, "Voice Services and Aviation Data Networks", 5th International Conference on Digital Avionics Systems, Dallas, TX, (2007) October 21-25.

\section{Authors}

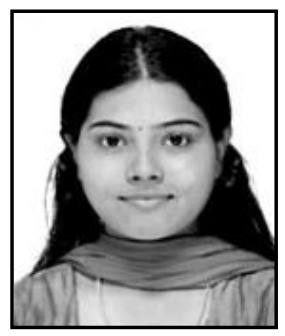

Amrutha, received her graduation (B.Tech) degree from Amrita University, Tamil Nadu in 2012. She is currently pursuing M.Tech degree in I.T(Networking) from Vellore Institute of Technology (VIT).Her areas of interest includes Cloud Computing andVoIP.

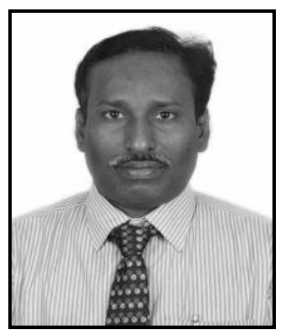

R. Thandeeswaran is a research scholar cum Associate Professor at VIT University, Vellore, India. He received his B.E. in Computer Engineering and M.Tech. (CSE) from Madurai Kamaraj and VIT University respectively. He published his research articles in international journals and conferences. He received active researcher award from VIT University, in the year 2013. He is life member of Computer Society of India. His areas of interest include Computer Networks and Software Engineering.

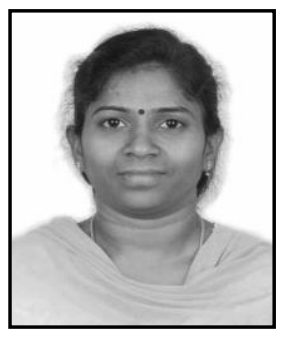

N. Jeyanthi is an Associate Professor in School of Information Technology and Engineering, VIT University, India. She received her $\mathrm{Ph} . \mathrm{D}$. and M.Tech. in Information Technology with Networking as specialization from VIT University, India in 2013 and 2006 respectively and BE in Computer Science and Engineering from Madurai Kamaraj University, Madurai, India in 1999. Her research interest is on Cloud network security real-time applications. She published twenty international journal papers and many international conference papers.

She is in the editorial board of international journals and acted as programme chair in many international conferences. She is a life member of Indian Society of Technical Education. 